\title{
Multiple Space Occupying Lesions in the Spleen: A Diagnostic Dilemma
}

Pramoda Koduru ${ }^{1}$, Uma Kundu ${ }^{2}$ and Manoop S Bhutani ${ }^{{ }^{*}}$

${ }^{1}$ Department of Gastroenterology, Hepatology and Nutrition, UT MD Anderson Cancer Center, Houston, Texas, USA

${ }^{2}$ Department of Pathology, UT MD Anderson Cancer Center, Houston, Texas, USA

*Corresponding author: Manoop S Bhutani, Director of Endoscopic Research and Development, Department of Gastroenterology, Hepatology and Nutrition-Unit 1466, UT MD Anderson Cancer Center, 1515 Holcombe Blvd., Houston, Texas, USA, Tel: 713-794-5073; Fax: 713-563-4398; E-mail: manoop.bhutani@mdanderson.org

Received date: Aug 28, 2015; Accepted date: Nov 24, 2015; Published date: Nov 26, 2015

Copyright: (C) 2015 Koduru P, et al. This is an open-access article distributed under the terms of the Creative Commons Attribution License, which permits unrestricted use, distribution, and reproduction in any medium, provided the original author and source are credited.

Keywords: Spleen; Histiocytes; Saroidosis

\section{Case}

A 56 year old woman presented to an outside ER for right upper quadrant pain that eventually resolved. CT scan of the abdomen done during that visit was read as showing multiple solid low density lesions in the spleen suspicious for metastases (Figure 1). Our radiologist's interpretation of the CT done in the ER was that these are most likely littoral cell angiomas. Other possibilities were sarcoidosis and splenic lymphoma. A percutaneous image guided fine-needle aspiration and core biopsy of one of the splenic lesions was performed revealing clusters of epithelioid histiocytes forming non-caseating granulomas (Figure 2) and numerous multinucleated giant cells (Figure 3, arrow) consistent with splenic sarcoidosis. There were no acid fast bacilli or fungi on special stains. The patient had a history of pulmonary sarcoidosis.

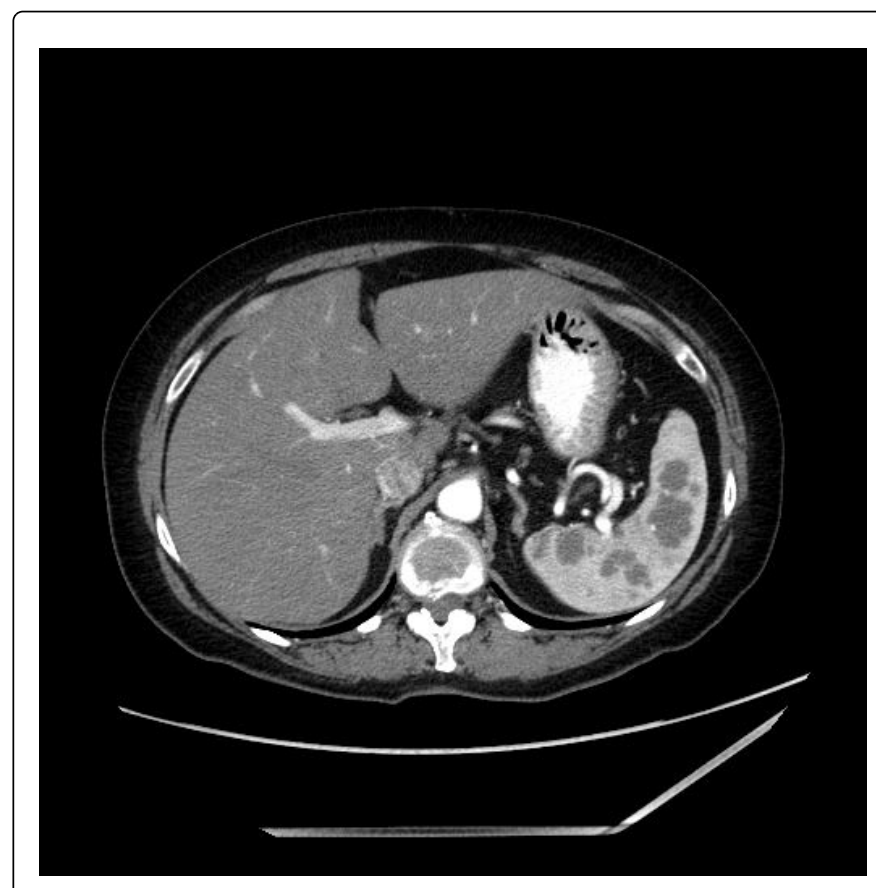

Figure 1: CT scan of the abdomen showing multiple solid low density lesions in the spleen suspicious for metastases.

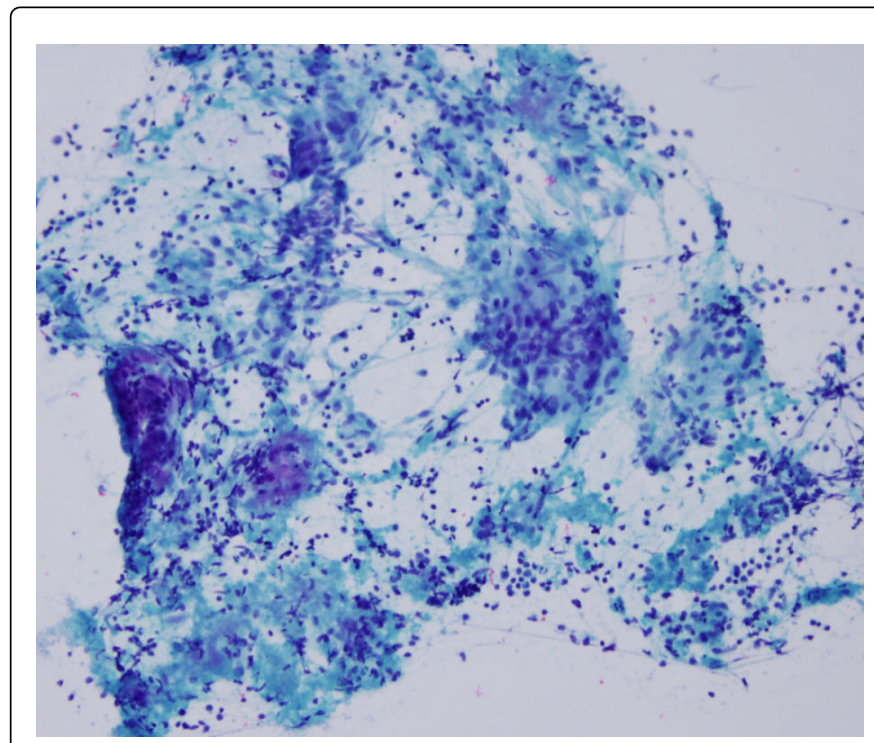

Figure 2: A percutaneous image guided fine-needle aspiration and core biopsy of splenic lesions revealing clusters of epithelioid histiocytes forming non-caseating granulomas.

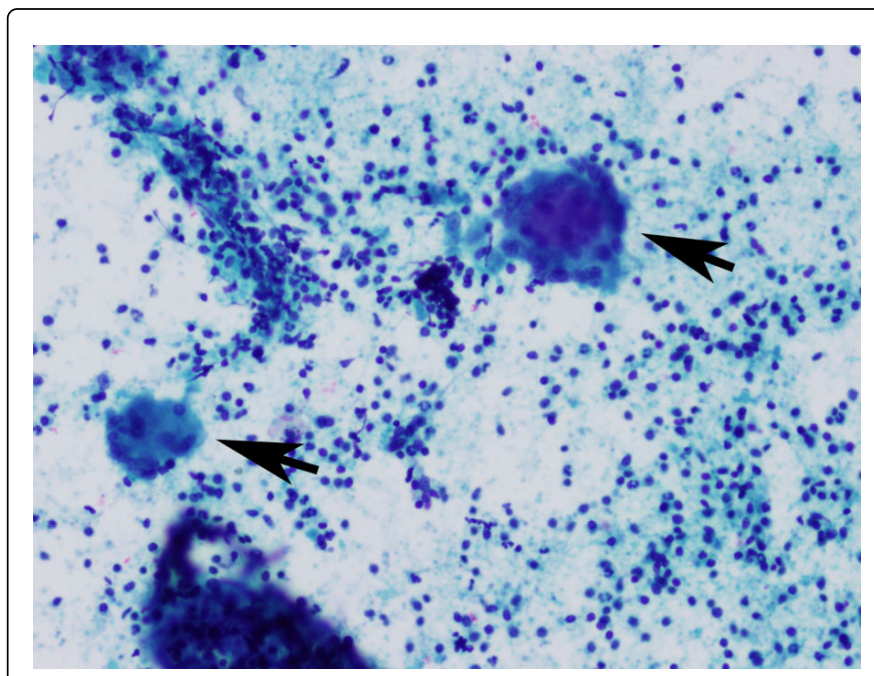

Figure 3: A percutaneous image guided fine-needle aspiration and core biopsy of splenic lesions revealing numerous multinucleated giant cells (arrows). 
The causes for multiple splenic lesions may include infections, inflammatory processes, primary neoplasms, metastases, vascular processes (e.g. infarcts) and systemic illness [1]. The main differential diagnoses in our patient included sarcoidosis, lymphoma, littoral cell angiomas or metastases. Malignant lymphoma affecting the spleen is mainly via secondary involvement and rarely $(<1 \%)$ spleen can be the site of primary involvement. Splenic metastases were considered high on differential by outside facility's radiologists but splenic metastases occur very rarely. Littoral cell angioma (LCA) is a benign tumor arising from littoral cells lining the red pulp sinuses of spleen. LCAs are mostly discovered incidentally on imaging but $50 \%$ of them may have splenomegaly or symptoms of hypersplenism. With non-specific imaging findings like heterogeneous echogenicity, LCA mimics benign or malignant neoplasms and disseminated infections. LCA occurs in association with immune-mediated diseases or visceral tumors in onethird of cases. Littoral cell angioma has also been reported in a patient with pulmonary sarcoidosis [1].

Splenic involvement of sarcoidosis occurs in $7 \%$ of patients [2]. Splenomegaly is the most common finding seen in one-third of cases but our patient did not have this manifestation. Most of the patients are asymptomatic but may rarely present with symptoms like abdominal discomfort from marked splenomegaly or symptoms of hypersplenism (anemia, leucopenia and thrombocytopenia). On imaging, splenic sarcoidosis presents in 2 forms: splenomegaly (most common) and multiple nodules. The nodular form has multiple, hypodense lesions ranging in size from few millimeters to $3 \mathrm{~cm}$, hypovascular with minimal or delayed enhancement. Imaging findings overlap with lymphoma but features like larger nodes being involved, greater tendency for confluence and retrocrural nodal involvement may suggest lymphoma over sarcoidosis. Splenic sarcoidosis may mimick neoplasm with atypical imaging features like capsular retraction which are seen mostly in neoplastic disease [3]. Diagnosis without clinical data based on imaging may be impossible at times as there is significant overlap of imaging features in different lesions [4]. Splenic biopsy if needed as in our case can be performed to resolve this diagnostic dilemma and this may avoid unnecessary splenectomy due to uncertain diagnosis.

\section{References}

1. Cordesmeyer S, Putzler M, Titze U, Paulus H, Hoffmann MW (2011) Littoral cell angioma of the spleen in a patient with previous pulmonary sarcoidosis: a TNF-alpha related pathogenesis? World J Surg Oncol 9: 106.

2. Warshauer DM (2007) Splenic sarcoidosis. Semin Ultrasound CT MR 28: 21-27.

3. Bauones S, Le Corroller T, Durieux O, Guenoun D, Grande JD, et al. (2014) Splenic sarcoidosis mimicking neoplastic disease. J Clin Ultrasound 42: 38-41.

4. Kamaya A, Weinstein S, Desser TS (2006) Multiple lesions of the spleen: differential diagnosis of cystic and solid lesions. Semin Ultrasound CT MR 27: 389-403.
This article was originally published in a special issue, entitled:

"Cytopathology", Edited by Borislav A. Alexiev, University of Maryland Medical Center, USA 